\title{
A CERTAIN SUBCLASS OF MULTIVALENT ANALYTIC FUNCTIONS WITH NEGATIVE COEFFICIENTS FOR OPERATOR ON HILBERT SPACE
}

\author{
Abbas Kareem Wanas \\ Department of Mathematics \\ College of Computer Science and Mathematics \\ University of Al-Qadisiya Diwaniya - Iraq \\ abbas.alshareefi@yahoo.co.uk
}

\begin{abstract}
By making use of the operators on Hilbert space, we introduce and study a subclass $\mathcal{A} k_{p}(\alpha, \beta, \delta, T)$ of multivalent analytic functions with negative coefficients. Also we obtain some geometric properties.

Key words: Hilbert space, analytic function, convex set, extreme points.
\end{abstract}

Abstrak. Dengan menggunakan operator-operator di ruang Hilbert, kami memperkenalkan dan mempelajari suatu subclass dari fungsi analitik multivalen $\mathcal{A} k_{p}(\alpha, \beta, \delta, T)$ dengan koefisien negarif. Kami juga mendapatkan beberapa sifat geometri mereka.

Kata kunci: Ruang Hilbert, fungsi analitik, himpunan konveks, titik-titik ekstrim.

\section{INTRODUCTION}

Let $\mathcal{A}_{p}$ be the class of functions $f$ of the form:

$$
f(z)=z^{p}+\sum_{n=1}^{\infty} a_{n+p} z^{n+p}(p \in \mathbb{N}=\{1,2, \ldots\}),
$$

which are analytic and $p$-valent in the open unit disk $U=\{z \in \mathbb{C}:|z|<1\}$. Let $k_{p}$ denote the subclass of $\mathcal{A}_{p}$ consisting of functions of the form:

$$
f(z)=z^{p}-\sum_{n=1}^{\infty} a_{n+p} z^{n+p}\left(a_{n+p} \geq 0, p \in \mathbb{N}=\{1,2, \ldots\}\right),
$$

2000 Mathematics Subject Classification: 30C45, 30C50.

Received: 06-06-2015, revised: 06-04-2015, accepted: 14-04-2015 
Definition 1.1. A function $f \in k_{p}$ is said to be in the class $\mathcal{A} k_{p}(\alpha, \beta, \delta)$ if it satisfies

$$
\left|\frac{f^{\prime}(z)-p z^{p-1}}{\alpha\left(f^{\prime}(z)-\beta\right)+p-\beta}\right|<0,
$$

where $0 \leq \alpha<1,0 \leq \beta<p, 0<\delta \leq 1$ dan $z \in U$.

Let $H$ be a Hilbert space on the complex field. Let $T$ be a linear operator on $H$. For a complex analytic function $f$ on the unit disk $U$, we denoted $f(T)$, the operator on $H$ defined by the usual Riesz-Dunford integral [2]

$$
f(T)=\frac{1}{2 \pi i} \int_{c} f(z)(z I-T)^{-1} d z
$$

where $I$ is the identity operator on $H, c$ is a positively oriented simple closed rectifiable contour lying in $U$ and containing the spectrum $\sigma(T)$ of $T$ in its interior domain [3]. Also $f(T)$ can be defined by the series

$$
f(T)=\sum_{n=0}^{\infty} \frac{f^{(n)}(0)}{n !} T^{n},
$$

which converges in the norm topology [4].

Definition 1.2. Let $H$ be a Hilbert space and $T$ be an operator on $H$ such that $T \neq \emptyset$ and $\|T\|<1$. Let $\alpha, \beta$ be real numbers such that $0 \leq \alpha<1,0 \leq \beta<p$, $0<\delta \leq 1$. An analytic function $f$ on the unit disk is said to belong to the class $\mathcal{A} k_{p}(\alpha, \beta, \delta, T)$ if it satisfy the inequality

$$
\left\|f^{\prime}(T)-p T^{p-1}\right\|<\delta\left\|\alpha\left(f^{\prime}(T)-\beta\right)+p-\beta\right\|,
$$

where $\emptyset$ denote the zero operator on $H$.

The operator on Hilbert space were consider recently be Xiaopei [8], Joshi [6], Chrakim et al. [1], Ghanim and Darus [5], and Selvaraj et al. [7].

\section{Main Results}

Theorem 2.1. Let $f \in k_{p}$ be defined by (2). Then $f \in \mathcal{A} k_{p}(\alpha, \beta, \delta, T)$ for all $T \neq \emptyset$ if and only if

$$
\sum_{n=1}^{\infty}(n+p)(1+\delta \alpha) a_{n+p} \leq \delta(p-\beta)(1+\alpha) .
$$

where $0 \leq \alpha<1,0 \leq \beta<p, 0<\delta \leq 1$.

The result is sharp for the function $f$ given by

$$
f(z)=z^{p}-\frac{\delta(p-\beta)(1+\alpha)}{(n+p)(1+\delta \alpha)} z^{n+p}, n \geq 1 .
$$


Proof. Suppose that the inequality (3) holds. Then, we have

$$
\begin{aligned}
\left\|f^{\prime}(T)-p T^{p-1}\right\| & -\delta\left\|\alpha\left(f^{\prime}(T)-\beta\right)+p-\beta\right\| \\
& =\left\|-\sum_{n=1}^{\infty}(n+p) a_{n+p} T^{n+p-1}\right\| \\
& -\delta\left\|\alpha p T^{p-1}-\sum_{n=1}^{\infty} \alpha(n+p) a_{n+p} T^{n+p-1}+p-\beta(1+\alpha)\right\| \\
& \leq \sum_{n=1}^{\infty}(n+p)(1+\delta \alpha) a_{n+p}-\delta(p-\beta)(a+\alpha) \leq 0 .
\end{aligned}
$$

Hence, $f \in \mathcal{A} k_{p}(\alpha, \beta, \delta, T)$.

To show the converse, let $f \in \mathcal{A} k_{p}(\alpha, \beta, \delta, T)$. Then

$$
\left\|f^{\prime}(T)-p T^{p-1}\right\|<\delta\left\|\alpha\left(f^{\prime}(T)-\beta\right)+p-\beta\right\|,
$$

gives

$$
\begin{aligned}
\left\|-\sum_{n=1}^{\infty}(n+p) a_{n+p} T^{n+p-1}\right\| \\
<\delta\left\|\alpha p T^{p-1}-\sum_{n=1}^{\infty} \alpha(n+p) a_{n+p} T^{n+p-1}+p-\beta(1+\alpha)\right\|
\end{aligned}
$$

Setting $T=r I(0<r<1)$ in the above inequality, we get

$$
\frac{\sum_{n=1}^{\infty}(n+p) a_{n+p} r^{n+p-1}}{\alpha p r^{p-1}-\sum_{n=1}^{\infty} \alpha(n+p) a_{n+p} r^{n+p-1}+p-\beta(1+\alpha)}<\delta
$$

Upon clearing denominator in (5) and letting $r \rightarrow 1$, we obtain

$$
\sum_{n=1}^{\infty}(n+p) a_{n+p}<\delta(p-\beta)(1+\alpha)-\sum_{n=1}^{\infty} \delta \alpha(n+p) a_{n+p} .
$$

Thus

which completes the proof.

$$
\sum_{n=1}^{\infty}(n+p)(1+\delta \alpha) a_{n+p} \leq \delta(p-\beta)(1+\alpha)
$$

Corollary 2.2. If $f \in \mathcal{A} k_{p}(\alpha, \beta, \delta, T)$, then

$$
a_{n+p} \leq \frac{\delta(p-\beta)(1+\alpha)}{(n+p)(1+\delta \alpha)}, n \geq 1 .
$$

Theorem 2.3. If $f \in \mathcal{A} k_{p}(\alpha, \beta, \delta, T)$ and $\|T\|<1, T \neq \emptyset$, then

$$
\|T\|^{p}-\frac{\delta(p-\beta)(1+\alpha)}{(p+1)(1+\delta \alpha)}\|T\|^{p+1} \leq\|f(T)\| \leq\|T\|^{p}+\frac{\delta(p-\beta)(1+\alpha)}{(p+1)(1+\delta \alpha)}\|T\|^{p+1}
$$


and

$$
p\|T\|^{p-1}-\frac{\delta(p-\beta)(1+\alpha)}{1+\delta \alpha}\|T\|^{p} \leq\left\|f^{\prime}(T)\right\| \leq p\|T\|^{p-1}+\frac{\delta(p-\beta)(1+\alpha)}{1+\delta \alpha}\|T\|^{p}
$$

The result is sharp for the function $f$ given by

$$
f(z)=z^{p}-\frac{\delta(p-\beta)(1+\alpha)}{(p+1)(1+\delta \alpha)} z^{p+1} .
$$

Proof. According to the Theorem 2.1, we get

$$
\sum_{n=1}^{\infty} a_{n+p} \leq \frac{\delta(p-\beta)(1+\alpha)}{(p+1)(1+\delta \alpha)} .
$$

Hence

$$
\begin{aligned}
\|f(T)\| & \geq\|T\|^{p}-\sum_{n=1}^{\infty} a_{n+p}\|T\|^{n+p} \\
& \geq\|T\|^{p}-\|T\|^{p+1} \sum_{n=1}^{\infty} a_{n+p} \\
& \geq\|T\|^{p}-\frac{\delta(p-\beta)(1+\alpha)}{(p+1)(1+\delta \alpha)}\|T\|^{p+1} .
\end{aligned}
$$

Also,

$$
\begin{aligned}
\|f(T)\| & \leq\|T\|^{p}+\sum_{n=1}^{\infty} a_{n+p}\|T\|^{n+p} \\
& \leq\|T\|^{p}+\frac{\delta(p-\beta)(1+\alpha)}{(p+1)(1+\delta \alpha)}\|T\|^{p+1}
\end{aligned}
$$

In view of Theorem 2.1, we have

$$
\sum_{n=1}^{\infty}(n+p) a_{n+p} \leq \frac{\delta(p-\beta)(1+\alpha)}{1+\delta \alpha} .
$$

Thus

$$
\begin{aligned}
\left\|f^{\prime}(T)\right\| & \geq p\|T\|^{p-1}-\sum_{n=1}^{\infty}(n+p) a_{n+p}\|T\|^{n+p-1} \\
& \geq p\|T\|^{p-1}-\|T\|^{p} \sum_{n=1}^{\infty}(n+p) a_{n+p} \\
& \geq p\|T\|^{p-1}-\frac{\delta(p-\beta)(1+\alpha)}{1+\delta \alpha}\|T\|^{p}
\end{aligned}
$$


and

$$
\begin{aligned}
\left\|f^{\prime}(T)\right\| & \leq p\|T\|^{p-1}+\|T\|^{p} \sum_{n=1}^{\infty}(n+p) a_{n+p} \\
& \leq p\|T\|^{p-1}+\frac{\delta(p-\beta)(1+\alpha)}{1+\delta \alpha}\|T\|^{p}
\end{aligned}
$$

Therefore the proof is complete.

Theorem 2.4. Let $f_{0}(z)=z^{p}$ and

$$
f_{n}(z)=z^{p}-\frac{\delta(p-\beta)(1+\alpha)}{(n+p)(1+\delta \alpha)} z^{n+p}, n \geq 1 .
$$

Then $f \in \mathcal{A} k_{p}(\alpha, \beta, \delta, T)$ if and only if it can be expressed in the form

$$
f(z)=\sum_{n=0}^{\infty} \lambda_{n} f_{n}(z)
$$

where $\lambda_{n} \geq 0$ and $\sum_{n=0}^{\infty} \lambda_{n}=1$.

Proof. Assume that $f$ can be expressed by (6). Then, we have

$$
f(z)=\sum_{n=0}^{\infty} \lambda_{n} f_{n}(z)=z^{p}-\sum_{n=0}^{\infty} \frac{\delta(p-\beta)(1+\alpha)}{(n+p)(1+\delta \alpha)} \lambda_{n} z^{n+p} .
$$

Thus

$$
\sum_{n=0}^{\infty} \frac{(n+p)(1+\delta \alpha)}{\delta(p-\beta)(1+\alpha)} \frac{\delta(p-\beta)(1+\alpha)}{(n+p)(1+\delta \alpha)} \lambda_{n}=\sum_{n=0}^{\infty} \lambda_{n}=1-\lambda_{0} \leq 1
$$

and so $f \in \mathcal{A} k_{p}(\alpha, \beta, \delta, T)$.

Conversely, suppose that $f$ given by (2) in in the class $\mathcal{A} k_{p}(\alpha, \beta, \delta, T)$. Then by Corollary 2.2, we have

$$
a_{n+p} \leq \frac{\delta(p-\beta)(1+\alpha)}{(n+p)(1+\delta \alpha)}
$$

Setting

$$
\lambda_{n}=\frac{(n+p)(1+\delta \alpha)}{\delta(p-\beta)(1+\alpha)} a_{n}, n \geq 1
$$

and $\lambda_{0}=1-\sum_{n=1}^{\infty} \lambda_{n}$. Then

$$
f(z)=\sum_{n=0}^{\infty} \lambda_{n} f_{n}(z)
$$

This completes the proof of the theorem.

Theorem 2.5. The class $\mathcal{A} k_{p}(\alpha, \beta, \delta, T)$ is a convex set. 
Proof. Let $f_{1}$ and $f_{2}$ be the arbitrary elements of $\mathcal{A} k_{p}(\alpha, \beta, \delta, T)$. Then for every $t(0 \leq t \leq 1)$, we show that $(1-t) f_{1}+t f_{2} \in \mathcal{A} k_{p}(\alpha, \beta, \delta, T)$. Thus, we have

$$
(1-t) f_{1}+t f_{2}=z^{p}-\sum_{n=1}^{\infty}\left((1-t) a_{n+p}+t b_{n+p}\right) z^{n+p} .
$$

Hence,

$$
\begin{aligned}
& \sum_{n=1}^{\infty}(n+p)(1+\delta \alpha)\left((1-t) a_{n+p}+t b_{n+p}\right) \\
& =(1-t) \sum_{n=1}^{\infty}(n+p)(1+\delta \alpha) a_{n+p}+t \sum_{n=1}^{\infty}(n+p)(1+\delta \alpha) b_{n+p} \\
& \leq(1-t) \delta(p-\beta)(1+\alpha)+t \delta(p-\beta)(1+\alpha) .
\end{aligned}
$$

This completes the proof.

\section{REFERENCES}

[1] Chrakim, Y., Lee, J.S., and Lee, S.H., "A certain subclass of analytic functions with negative coefficients for operators on Hilbert space", Math. Japonica, 47(1) (1998), 155-124.

[2] Dunford, N., and Schwarz, J.T., Linear Operator, Part I, General Theory, New York London, Inter Science, 1958.

[3] Fan, K., "Analytic functions of a proper contraction", Math. Z., 160 (1978), 275-290.

[4] Fan, K., "Julia's lemma for operators", Math. Ann., 239 (1979), 241-245.

[5] Ghanim, F., and Darus, M., "On new subclass of analytic p-valent function with negative coefficients for operators on Hilbert space", Int. Math. Forum, 3:2 (2008), 69-77.

[6] Joshi, S.B., "On a class of analytic functions with negative coefficients for operators on Hilbert Space", J. Appr. Theory and Appl., (1998), 107-112.

[7] Selvaraj, C., Pamela, A.J., and Thirucheran, M., "On a subclass of multivalent analytic functions with negative coefficients for contraction operators on Hilbert space", Int. J. Contemp. Math. Sci., 4:9 (2009), 447-456.

[8] Xiapei, Y., "A subclass of analytic p-valent functions for operator on Hilbert Space", Math. Japonica , 40:2 (1994), 303-308. 